Communications in Physics, Vol.20, No. 1 (2010), pp. 45-50

\title{
NONLINEAR COUPLER FOR OPTICAL FIBER MACH-ZEHNDER INTERFEROMETER
}

\author{
HO QUANG QUY \\ Academy of Military Science and Technology \\ VU NGOC SAU \\ Vinh University \\ NGUYEN THI THANH TAM \\ QuangNam University \\ NGUYEN VAN HOA \\ Hong Duc University
}

\begin{abstract}
In this article the equations for two waves propagating in two fibers of nonlinear Kerr coupler for optical Mach-Zehnder interferometer are derived. Based on the resolution of those equations, the expressions describing relation between coherent length, input intensity of the wave, nonlinear coefficient of refractive index for $3 d B$ Kerr coupler are introduced and discussed.
\end{abstract}

\section{INTRODUCTION}

The optical fiber Mach-Zehnder interferometer (OFMZI) consists of three components: two couplers and one pair of fibers (see Fig.1) $[1,2]$.
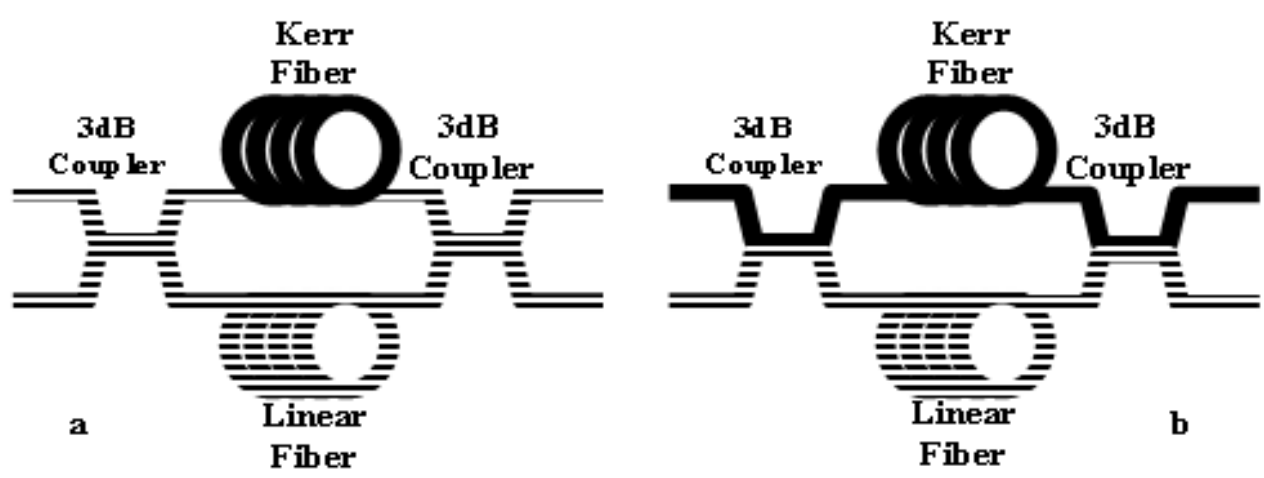

Fig. 1. OFMZI with two linear couplers (a) and with two nonlinear couplers (b) 
The core of one of fibers is nonlinear Kerr medium. Two couplers are $3 \mathrm{~dB}$ transfer, i.e., the ratio of output intensity and input intensity is 50 percent [3, 4]. Really, two couplers consist from two linear fibers of "3dB" length. But in this way, the designing process of OFMZI becomes complex and leads to the reduction of the transfer efficiency, caused by connecting and end-fire coupling [5]. To avoid this complexity we propose using one Kerr fiber to produce the coupler for OFMZI (Fig.1b). In this way two linear couplers are replaced by two nonlinear couplers.

To have useful parameters of coupler for designing OFMZI in the furture, in this work we investigate the conditions of Kerr fiber in order to have $3 \mathrm{~dB}$ coupler by solving wave equations, which describe the propagation of two waves and intensity transfer process $[6]$.

\section{WAVE EQUATIONS IN NONLINEAR COUPLER}

Consider a wave with complex amplitude:

$$
E(x, y, z, t)=\sum_{i} A_{i}(z) \phi_{i}(x, y) \exp \left[i\left(\omega t-\beta_{i} z\right)\right],
$$

where $\phi_{i}(x, y)$ is transverse space mode of $\mathrm{i}$-field; $\beta_{i}$ is the propagating coefficient; $A_{i}(z)$ is the amplitude, propagates in the nonlinear coupler with refractive indexes given by:

$$
\begin{aligned}
& n_{1}^{2}(x, y)=n_{s}^{2}(x, y)+\Delta n_{1}^{2}(x, y)+n_{n l}^{2}|E(x, y)|^{4} \\
& n_{2}^{2}(x, y)=n_{s}^{2}(x, y)+\Delta n_{2}^{2}(x, y)
\end{aligned}
$$

where $n_{s}$ is the refractive index of clad; $\Delta n_{1}$ is the linear refractive index of core of Kerr fiber; $n_{n l}$ is the nonlinear refractive coefficient of Kerr fiber; $\Delta n_{2}$ is the refractive index of core of linear fiber.

Two waves in coupler satisfy following equation:

$$
\left(\Delta_{T}+\omega^{2} \mu \epsilon(x, y)-\beta_{i}^{2}\right) E_{i}(x, y)=-\mu \delta \epsilon(x, y) E_{i}(x, y) .
$$

Substituting (1), (2) into (3), using slowly varying approximation and after some arrangement we have:

$$
\begin{aligned}
& \frac{d A_{1}(z)}{d z}=-i C_{11} A_{1}(z)-i C_{n l} A_{1}(z)-i C_{12} A_{2}(z) \exp \left[i\left(\beta_{1}-\beta_{2}\right) z\right], \\
& \frac{d A_{2}(z)}{d z}=-i C_{22} A_{2}(z)-i C_{21} A_{1}(z) \exp \left[-i\left(\beta_{1}-\beta_{2}\right) z\right],
\end{aligned}
$$

where $C_{i j}=\frac{\omega \epsilon_{0}}{4} \iint \phi_{i}^{*}(x, y) \Delta n_{i}^{2} \phi_{j}(x, y) d x d y$ describes the coupling of two waves in fiber number i; $C_{i i}=\frac{\omega \epsilon_{0}}{4} \iint \phi_{i}^{*}(x, y) \Delta n_{j}^{2} \phi_{i}(x, y) d x d y$ describes the noise from neighbor fiber; $C_{n l}=\frac{\omega \epsilon_{0}}{4} \iint \phi_{i}^{*}(x, y) n_{n l}^{2}|E(x, y)|^{4} \phi_{i}(x, y) d x d y$ describes influence of Kerr effect in nonlinear fiber.

In the fiber coupler, it is real that $n_{n l}<<\Delta n_{1}$ and $n_{n l}<<\Delta n_{2}$, so $C_{n l}$ slowly varies with changing of amplitude $A_{1}$. From that we can consider:

$$
C_{n l} \approx \frac{\omega \epsilon_{0} n_{n l}^{2} I_{i n}^{2}}{4} \approx \text { const. }
$$


From (4) we can see that coefficients $C_{11}, C_{22}, C_{n l}$ make the propagating coefficient $\beta_{i}$ to change.

If $A_{1}(z) \exp \left(-i \beta_{1} z\right)$ and $A_{2}(z) \exp \left(-i \beta_{2} z\right)$ are roots of noiseless equation for the every fiber, we will have $\left(\frac{d A_{i}(z)}{d z}-\beta_{i}^{2}\right) E=0$. Then from (4) we can rewrite:

$$
\begin{aligned}
& \frac{d A_{1}^{\prime}(z)}{d z}=i C_{12} A_{2}^{\prime}(z) \exp [i(2 \Delta \beta) z] \\
& \frac{d A_{2}^{\prime}(z)}{d z}=-i C_{21} A_{1}^{\prime}(z) \exp [-i(2 \Delta \beta) z]
\end{aligned}
$$

with

$$
\begin{aligned}
& A_{1}(z)=A_{1}^{\prime}(z) \phi_{1}(x, y) \exp \left[-i\left(C_{11}+C_{n l}\right) z\right] \\
& A_{2}(z)=A_{2}^{\prime}(z) \phi_{2}(x, y) \exp \left[-i\left(C_{22}\right) z\right]
\end{aligned}
$$

and

$$
2 \Delta \beta=\left(\beta_{1}+C_{11}+C_{n l}\right)-\left(\beta_{2}+C_{22}\right)=\left(\beta_{1}-\beta_{2}+C_{11}-C_{22}\right)+C_{n l} .
$$

\section{POWER TRANSFER OF NONLINEAR COUPLER}

Solving (6) with considering $A_{1}(0)=A_{0}$ (input amplitude of wave in Kerr fiber) and $A_{2}(0)=A_{0}$ (input amplitude of wave in linear fiber) and we have:

$$
\begin{aligned}
& A_{1}^{\prime}(z)=A_{0} \exp (i \Delta \beta z) \cos \left(z \sqrt{(\Delta \beta)^{2}+C_{12} C_{21}}\right)-i \frac{(\Delta \beta) \sin \left(z \sqrt{(\Delta \beta)^{2}+C_{12} C_{21}}\right)}{\sqrt{(\Delta \beta)^{2}+C_{12} C_{21}}}, \\
& A_{2}^{\prime}(z)=i A_{0} \exp (i \Delta \beta z) C_{21} \frac{(\Delta \beta) \sin \left(z \sqrt{(\Delta \beta)^{2}+C_{12} C_{21}}\right)}{\sqrt{(\Delta \beta)^{2}+C_{12} C_{21}}} .
\end{aligned}
$$

Consider two fibers in coupler with same linearity, i.e. $C_{11}=C_{22}, C_{12}=C_{21}=C$ and there is a linear resonant coupling, i.e., $\beta_{1}=\beta_{2}$ (two waves propagate with same propagating coefficient in linear fiber), then we have $\Delta \beta=\frac{C_{n l}}{2}$ and

$$
\begin{aligned}
& A_{1}^{\prime}(z)=A_{0} \exp \left(\frac{i z C_{n l}}{2}\right) \cos \left(z \sqrt{\left(\frac{C_{n l}}{2}\right)^{2}+C^{2}}\right)-i \frac{\left(\frac{C_{n l}}{2}\right) \sin \left(z \sqrt{\left(\frac{C_{n l}}{2}\right)^{2}+C^{2}}\right)}{\sqrt{\left(\frac{C_{n l}}{2}\right)^{2}+C^{2}}} \\
& A_{2}^{\prime}(z)=i A_{0} \exp \left(i z \frac{C_{n l}}{2}\right) C_{21} \frac{\left(\frac{C_{n l}}{2}\right) \sin \left(z \sqrt{\left(\frac{C_{n l}}{2}\right)^{2}+C^{2}}\right)}{\sqrt{\left(\frac{C_{n l}}{2}\right)^{2}+C^{2}}} .
\end{aligned}
$$


The optical power of wave in fiber is given by:

$$
P(z) \cong\left|A^{\prime}(z)\right|^{2}
$$

Using (10) and substituting (5) into (9) we have expressions for transferring power between fiber in one nonlinear coupler as follows:

$$
\begin{aligned}
& \frac{P_{1}(z)}{P_{1}(0)}=1-\frac{C^{2}}{\frac{\omega^{2} \epsilon_{0}^{2} n_{n l}^{4} I_{i n}^{4}}{16}+C^{2}} \sin ^{2}\left(z \sqrt{\frac{\omega^{2} \epsilon_{0}^{2} n_{n l}^{4} I_{i n}^{4}}{16}+C^{2}}\right), \\
& \frac{P_{2}(z)}{P_{1}(0)}=\frac{C^{2}}{\frac{\omega^{2} \epsilon_{0}^{2} n_{n l}^{4} I_{i n}^{4}}{16}+C^{2}} \sin ^{2}\left(z \sqrt{\frac{\omega^{2} \epsilon_{0}^{2} n_{n l}^{4} I_{i n}^{4}}{16}+C^{2}}\right) .
\end{aligned}
$$

From (11) we can see a portion of power periodically transfer from one fiber into other (see Fig.2). For the linear coupler $\left(n_{n l}=0\right)$, the length $L_{c o h}=\frac{\pi}{2 C}$ is defined as coherent length, for which power in one fiber transfer completely into second one.
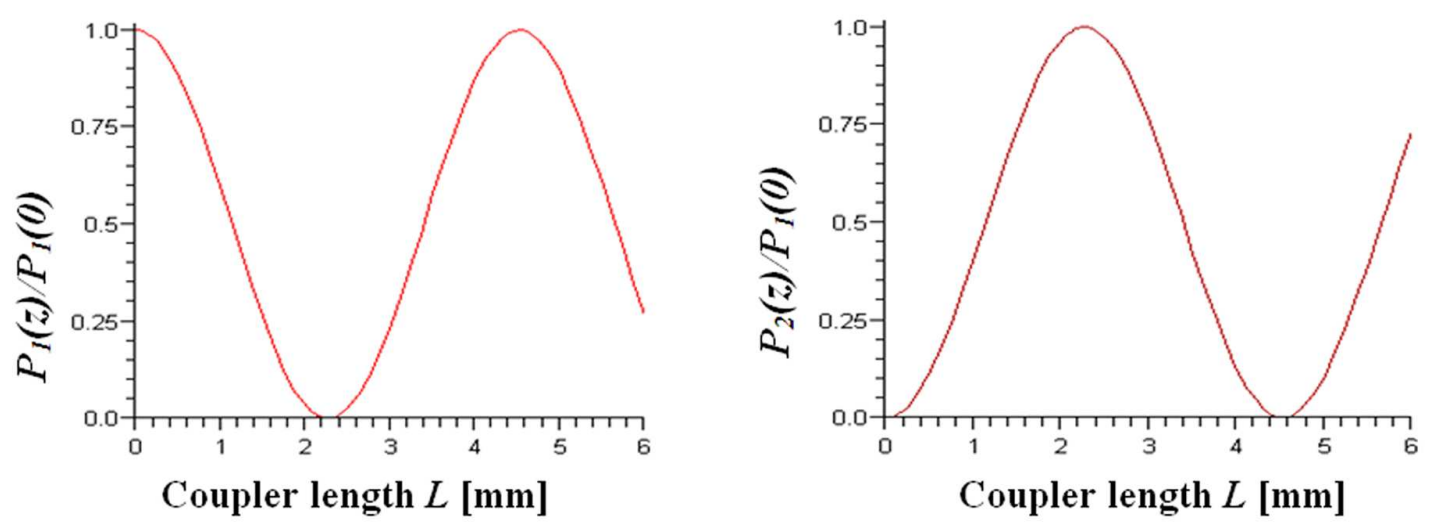

Fig. 2. Periodical transfer of power from one fiber to other of linear coupler: $\lambda=1.33 \mu \mathrm{m}, \mathrm{C}=0.694 / \mathrm{mm}[6], n_{n l}=0, \Delta \beta=0$.

The most useful of linear couplers is the " $3 \mathrm{~dB}$ " coupler, for which a half of power in one fiber transfers into second one if its length is a half of coherent length, i.e., $L_{3 d B}=\frac{L_{c o h}}{2}$.

But, for nonlinear coupler the coherent length is given by:

$$
L_{\text {cohnon }}=\frac{\arcsin \left(\sqrt{\frac{\frac{\omega^{2} \epsilon_{0}^{2} n_{n l}^{4} I_{i n}^{4}}{16}+C^{2}}{C^{2}}}\right)}{\sqrt{\frac{\omega^{2} \epsilon_{0}^{2} n_{n l}^{4} I_{i n}^{4}}{16}+C^{2}}},
$$

and " $3 \mathrm{~dB}$ " length is determined as follows: 


$$
L_{3 d \text { Bnon }}=\frac{\arcsin \left(\sqrt{\frac{\frac{\omega^{2} \epsilon_{0}^{2} n_{n l}^{4} I_{i n}^{4}}{16}+C^{2}}{2 C^{2}}}\right)}{\sqrt{\frac{\omega^{2} \epsilon_{0}^{2} n_{n l}^{4} I_{i n}^{4}}{16}+C^{2}}} .
$$

From (12) and (13) we can see that, the coherent length and "3dB" length of nonlinear Kerr coupler depend not only on coupling coefficient $\mathrm{C}$, but also on input intensity $I_{\text {in }}$ (see Fig. 3). Moreover, with suitable input intensity $\left(I_{i n}=1.510^{5} \mathrm{~W} / \mathrm{mm}^{2}\right.$ in our example) the coupler can have transfer coefficient 50 percent (see Fig. 3a). When the nonlinear refractive coefficient $n_{n l}$ or input intensity $I_{\text {in }}$ are too high $\left(>I_{\text {in }}=1.510^{5} \mathrm{~W} / \mathrm{mm}^{2}\right.$ in our example) the transfer coefficient never reaches 50 percent (see Fig. 3b).

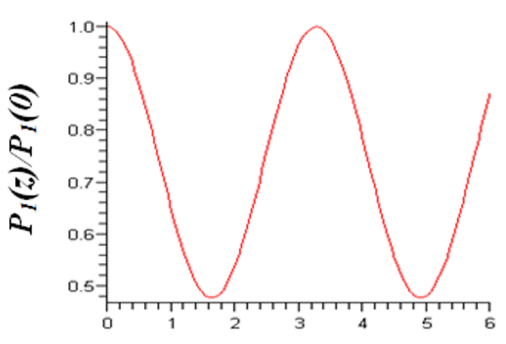

Coupler length $L[\mathrm{~mm}]$

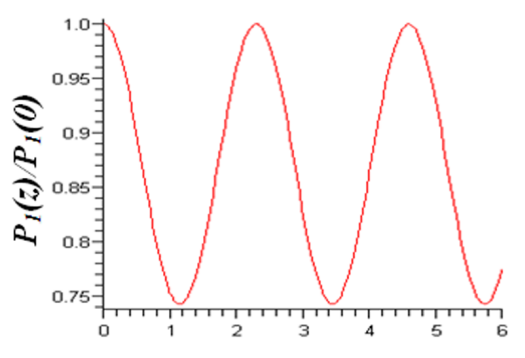

Coupler length $L[\mathrm{~mm}]$

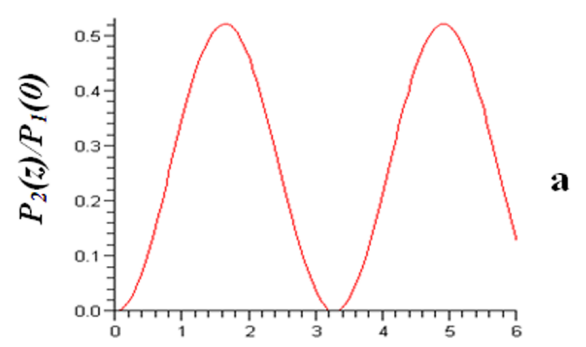

Coupler length $L[\mathrm{~mm}]$

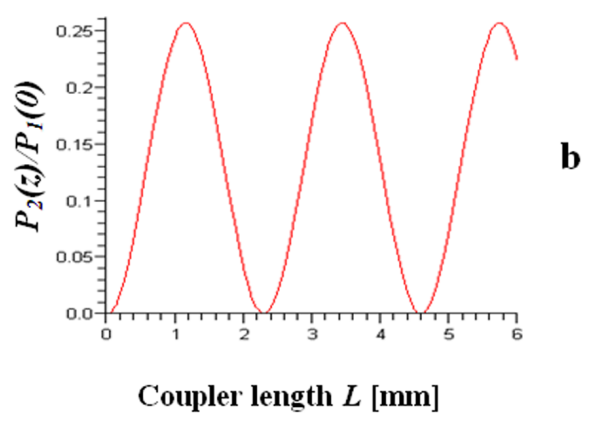

Fig. 3. Periodical transfer of power from one fiber to other of nonlinear coupler: $\lambda=1.33 \mu \mathrm{m}, \mathrm{C}=0.694 / \mathrm{mm}[6], n_{n l}=5.010^{-8} \mathrm{~mm}^{2} / W, \Delta \beta=0$, $I_{\text {in }}=1.510^{5} \mathrm{~W} / \mathrm{mm}^{2}(\mathrm{a})$ and $I_{\text {in }}=2.010^{5} \mathrm{~W} / \mathrm{mm}^{2}(\mathrm{~b})$.

\section{CONCLUSION}

The expressions for power transfer between fibers of nonlinear Kerr coupler are derived by wave equations with proposing Kerr effect is small as a noise. From those expressions the coherent length and " $3 \mathrm{~dB}$ " length of nonlinear coupler are found out. They depend on input intensity of wave and nonlinear coefficient of refractive index. In 
fact, the nonlinear " $3 \mathrm{~dB}$ " coupler can be designed with chosen qualities of Kerr fiber and power of input wave. With above characters, the OFMZI with nonlinear couplers will be seen as one with smooth mirrors (the reflectivity depends on input intensity). Moreover, the bistability of OFMZI depends on refractive index, length and nonlinear refractive coefficient of nonlinear coupler. The results of this work play an important role for designing the optical fiber Mach-Zehnder Interferometer, and also in the investigation of the bistable characteristics of it in the future.

\section{REFERENCES}

[1] M. Soljacic, Phys. Rev.E 66 (2002) 05560.

[2] T. Tanabe, Opt. Switching OPN (2005) 35.

[3] M. H. Till, Microwave and Optical Technology Letters, 31 (6) (2001) 411-415.

[4] R. Clarevo, Opt. Letter 30 (21) (2005) 2861-2863.

[5] G. Knoblauch, H.N. Toussaint, Telcom. report 6 (1983) 96.

[6] J. M. Jonathan, Doson (2004) 245.

Received 04 August 2008. 